\title{
Application of Communication Technology in the Distributed Network Sensor Based on Radio Frequency Chip RF
}

\author{
Qiuping $\mathrm{Wu}^{*}$
}

Chongqing Technical and Business Institute, Chongqing, China

\begin{abstract}
In this paper, the work of transmission test field data to the terminal, the wireless data transmission capacity in order to test the wireless data transmission terminal objective and effective, the choice of the open area and factory two site, to test the receiving terminal in different condition, respectively in the experiment of the terminal data transmission. The transmission distance, transmission rate and bit error rate were tested with satisfactory results. The experimental results are summarized, the increase of transmission distance, were reviewed and ideas to increase transmission range needs to expand its wireless network through a wireless communication base station, in the coverage of wireless communication base station, any wireless data transmission terminal can be effectively complete the data transmission between the host computer, and data exchange.
\end{abstract}

Keywords: Data fusion, distributed sensor network, middleware, mobile agent.

\section{INTRODUCTION}

With the rapid development of communication technology, embedded computing technology and sensor technology and increasingly sophisticated, with perceived competence, capability of computation and communication micro sensors began to appear in the world [1]. The distributed sensor network (DSN), which is composed of these sensors, has attracted much attention. On this scale, often contain thousands of nodes and long-term in the unattended state sensor network system in the field of military, environment, health, family and business, and has wide application prospect. At present, the U.S. government and foreign research institutions invested heavily, set up a lot of research programs on the DSN. The more important are: PicoRadio, WINS, Dust Smart, AMPS, SCADDS [2].

Wired sensor based on IEEE1451 standard and IEEE1451 and Bluetooth wireless sensor network protocol. The IEEE1451 network sensor represents the development direction of next generation sensor [3]. Now the design of wireless sensor network based on IEEE1451 standard with a special interface module and integrated chip such as ED1520, PLCC-44, STIM module and software module uses the standard IEEE1451 [4]. The sensor in a wireless network has been introduced Bluetooth technology hardware and software development platform based on EBDK system, such as the Bluetooth development of Ericsson, QSDK rapid development system of AD. The system can be convenient, fast development of radio transmitting and receiving module based on Bluetooth protocol (Y Zhao. et al, 1997). The development of sensor networks, the measurement and control system of active information processing and remote

*Address correspondence to this author at the Chongqing Technical and Business Institute, Chongqing, China; Tel: +86-153-1036-0588;

E-mail: 25107164@qq.com real-time measurement is possible. The development of wireless sensor network is still in its infancy, with the development needs of the full range of test parameters and the network technology, wireless sensor networks will become the hotspot of future research [5].

Based on the description of the concept of network sensor and IEEE1451 and basic content, proposed one kind based on the RF wireless communication technology wireless RF transceiver integrated chips to solve the wireless network sensor in the transmission requirement plan. The concrete work is as follows: on several mainstream wireless communication technologies are analyzed and compared, choose the RF transceiver integrated chips as the main object of study, in the NRF401 chip is studied, making the wireless data transmission terminal based on NRF401 chip, then debugging in the hardware and software of wireless data transceiver, data transceiver test were carried out in different working environments, the terminal in the process of data transmission, the transmission distance of the transmission rate and bit error rate and other indicators were tested.

\section{RELATED METHOD AND THEORY}

\subsection{Distributed Sensor Network Model Based on C/S}

A DSN consists of a series of sensor nodes, a series of processing units, and these units are connected to each other by communication network. A processing unit may be associated with one or more sensor connection [6]. A sensor can send the information to one or more processing units. A processing unit connected sensors associated with it as a sensor cluster [7]. The data from the sensor to the processing unit associated with it, data integration and fusion in there. The processing unit can coordinate with each other in order to obtain better estimates of the environment. Distributed sensor networks system was showed in Fig. (1). 


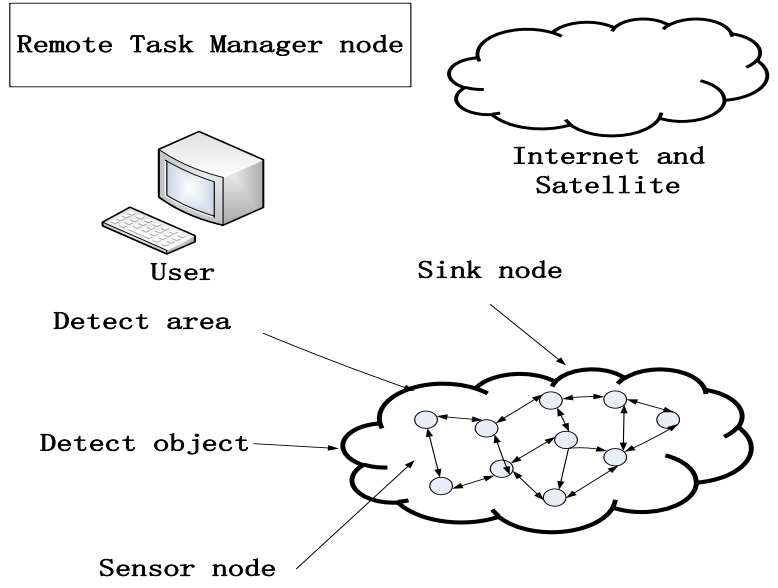

Fig. (1). Distributed sensor networks structure.

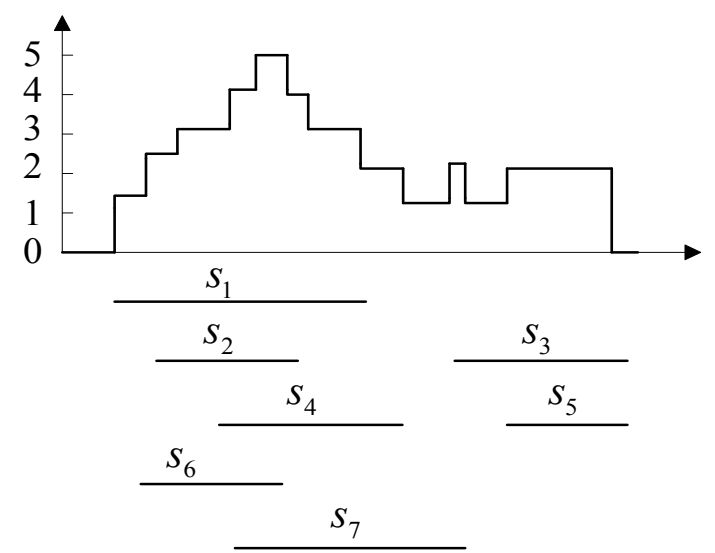

establish the overlap function

Fig. (2). The nodes with overlapping function to calculate the formation.

No matter what kind of distributed sensor network topological structure, data processing method at present is the use of the network model, namely the client/server model (CS model).

For n-dimensional objective optimization problem, the solution space is:

$S=\left\{X \mid X=\left(x_{1}, x_{2}, \cdots, x_{n}\right)^{T}\right\} \subseteq R^{n}$.

Initial population of SFLA has $\mathrm{F}$ frogs. The $\mathrm{F}$ frogs are sorted by the fitness values $f\left[X_{k}(t)\right]$. Each group frog number is N. Let $B_{j}$ be $j$ groups, there is:

$B_{j}=\left\{X_{j+M(l-1)}(t) \in P_{t} \mid 1 \leq l \leq N\right\}, 1 \leq j \leq M$

\subsection{Data Fusion Algorithm for Distributed Sensor Net- works}

We define the processing nodes some nodes and the node $\mathrm{S} 1, \ldots, \mathrm{Sn}$, is $\mathrm{P}$, the confidence interval of each node with the $\mathrm{Xj}$ said

$X_{j}(x)=\left\{\begin{array}{c}1, a_{j} \leq x \leq b_{j} \\ 0, x>b_{j}, x<a_{j}\end{array}\right.$
Fig. (2) shows a set consisting of seven nodes with overlapping function to calculate the formation. Therefore, the actual measured values shall be located in the overlapping function has the highest peak value and width of the most wide area.

Multi resolution analysis proposed a very efficient method to deal with overlapping functions, that is, first of all in a coarser resolution up processing overlapping functions, to identify the peak interval, thus narrowing the scope, and then only in the narrow range improve resolution again to deal with overlapping functions, repeat the process until you get let us satisfactory resolution of narrow peaks, and actual parameter values in the narrow peaks in the horizontal coverage area coordinate.

\section{EXPERIMENTAL RESULTS}

\subsection{The Design of Hardware}

In the sending state data when the microcontroller receives the low level signal button is pressed, the pin out of Gaoping into the sending State data [8]. At the same time random strobe and different crystal and frequency hopping between $433 \mathrm{M} \mathrm{Hz}$ and $434 \mathrm{M} \mathrm{Hz}$ in CS with the same frequency, transmission of data information of two at each 


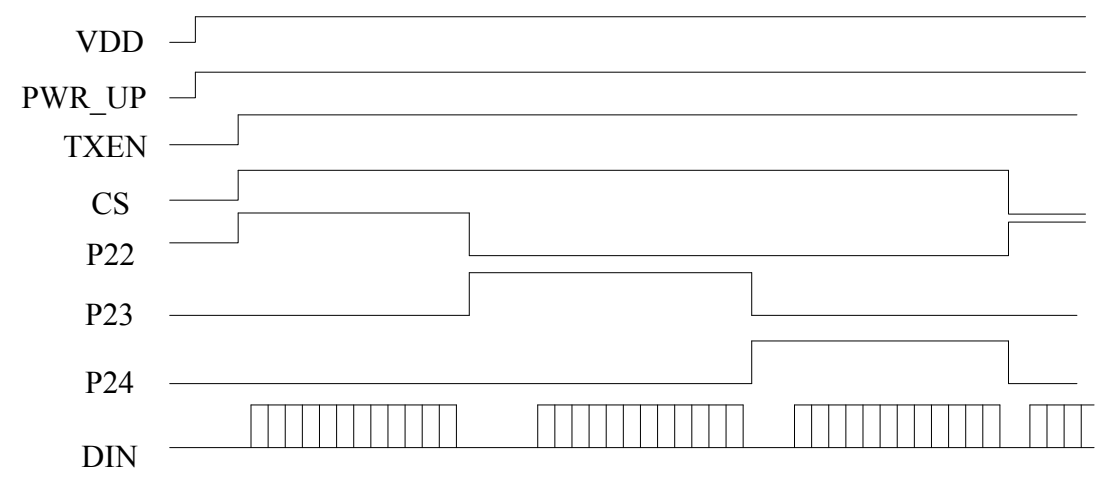

Data transmission timing diagram

Fig. (3). The data transmission time sequence.

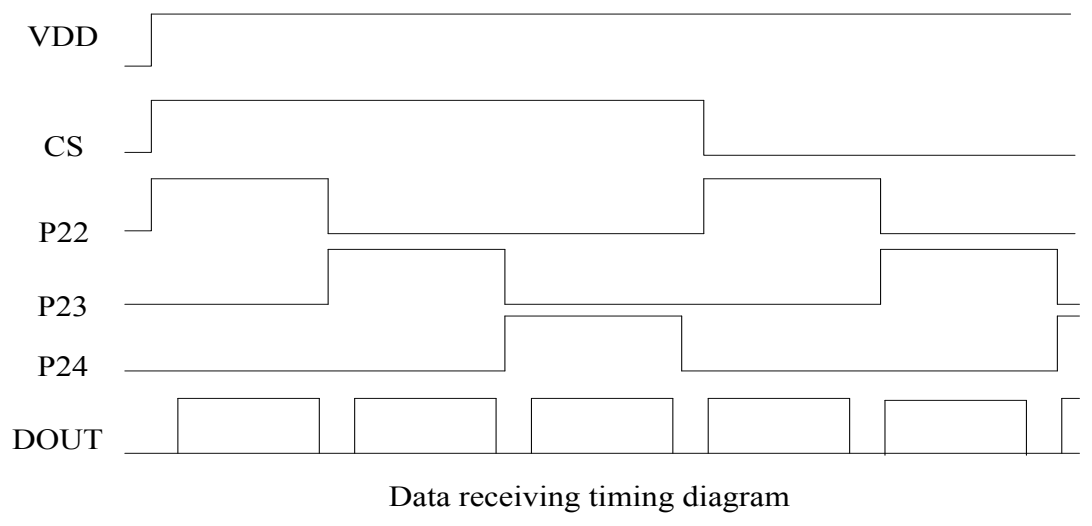

Fig. (4). Data receiving sequence.

frequency point, and then jump to the next frequency. The data transmission time sequence was shown in Fig. (3). Each of the receiving frequency under the working time is 7 times the frequency point of working time, in order to receive within each receiving frequency work hours to complete data transmission information. Data receiving sequence was shown in Fig. (4).

\subsection{Experiment and Analysis}

The mobile Agent system provides a new method for realizing the distributed data fusion. Since the new Agent can be injected into the existing networks, these networks can be re assigned tasks and set up in order to cope with the unpredictable changes in the environment [9-11]. Agent moves to a node in the network and uses the existing information on the node to fuse data.. When it finishes each node in the network and returns to the main node, the fusion process is finished. This method has many advantages, especially in wireless sensor networks, such as scalability, reliability, scalability, adaptability, low power consumption and accuracy. The network nodes randomly distributed was shown in Fig. (5).

In order to reduce the communication between this system from the implementation of the CSMA/CA protocol to communicate with the base station node, in the former, the first carrier monitoring in the communication channel, when monitoring the channel idle time is greater than the preset time slot, think that the channel is idle. If in the period of time to monitor the channels are occupied, then re monitor- ing, until the time to meet the conditions. But also can send the data immediately, but to adopt a "binary exponential backoff algorithm" random delay a backoff time, because at this time there may be more than one from the station waiting for the channel conflict free, the maximum probability. The CSMA/CA algorithm flow was shown in Fig. (6).

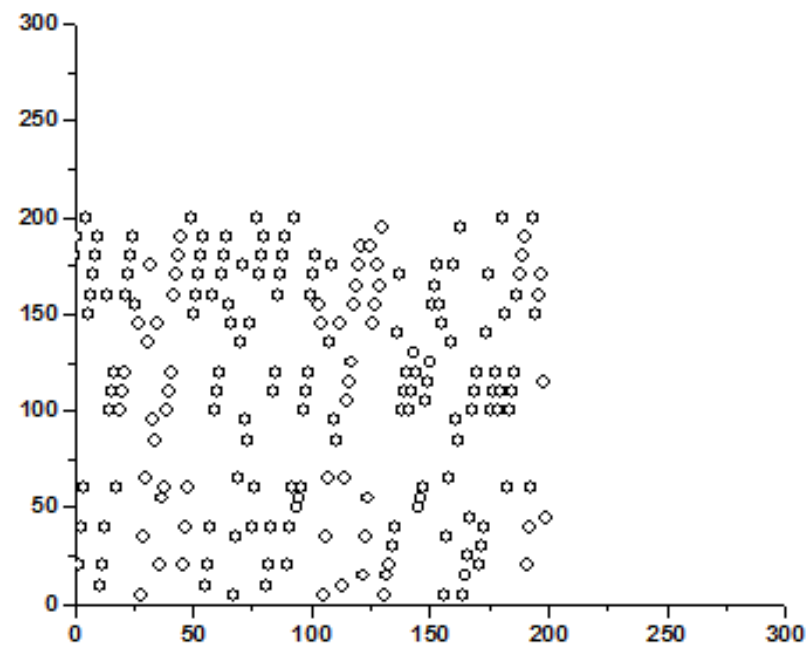

Fig. (5). Network nodes randomly distributed

The results of classified collection were shown in Table 
Table 1. Classified collection results.

\begin{tabular}{|c|c|c|c|c|c|c|}
\hline Time & 1 & 2 & 3 & 4 & 5 & 6 \\
\hline 10 & 93 & 95 & 91 & 91 & 91 & 93 \\
\hline 10 & 95 & 97 & 95 & 95 & 95 & 95 \\
\hline 15 & 89 & 95 & 97 & 97 & 97 & 97 \\
\hline 20 & 83 & 89 & 99 & 99 & 99 & 99 \\
\hline 25 & 95 & 93 & 95 & 93 & 83 & 97 \\
\hline 30 & 93 & 95 & 89 & 95 & 93 & 95 \\
\hline 35 & 95 & 97 & 93 & 97 & 95 & 89 \\
\hline 40 & 93 & 93 & 95 & 99 & 97 & 93 \\
\hline 45 & 93 & 95 & 93 & 93 & 99 & 95 \\
\hline 50 & 95 & 97 & 95 & 95 & 89 & 97 \\
\hline 55 & 97 & 99 & 97 & 97 & 83 & 99 \\
\hline 60 & 99 & 95 & 99 & 99 & 95 & 92 \\
\hline
\end{tabular}

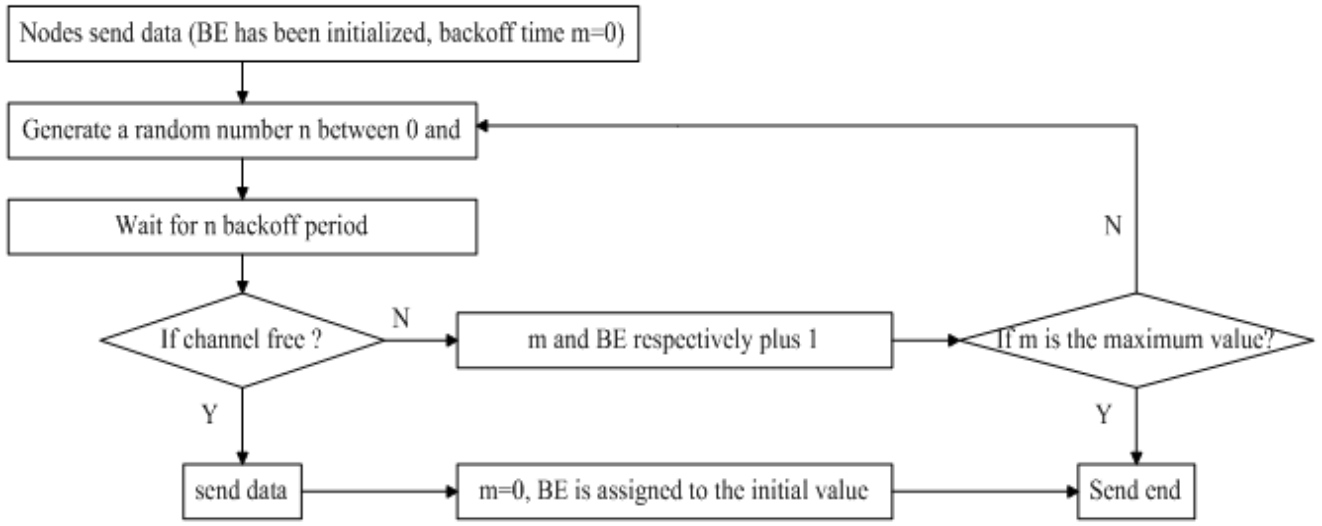

Fig. (6). The CSMA/CA algorithm flow.
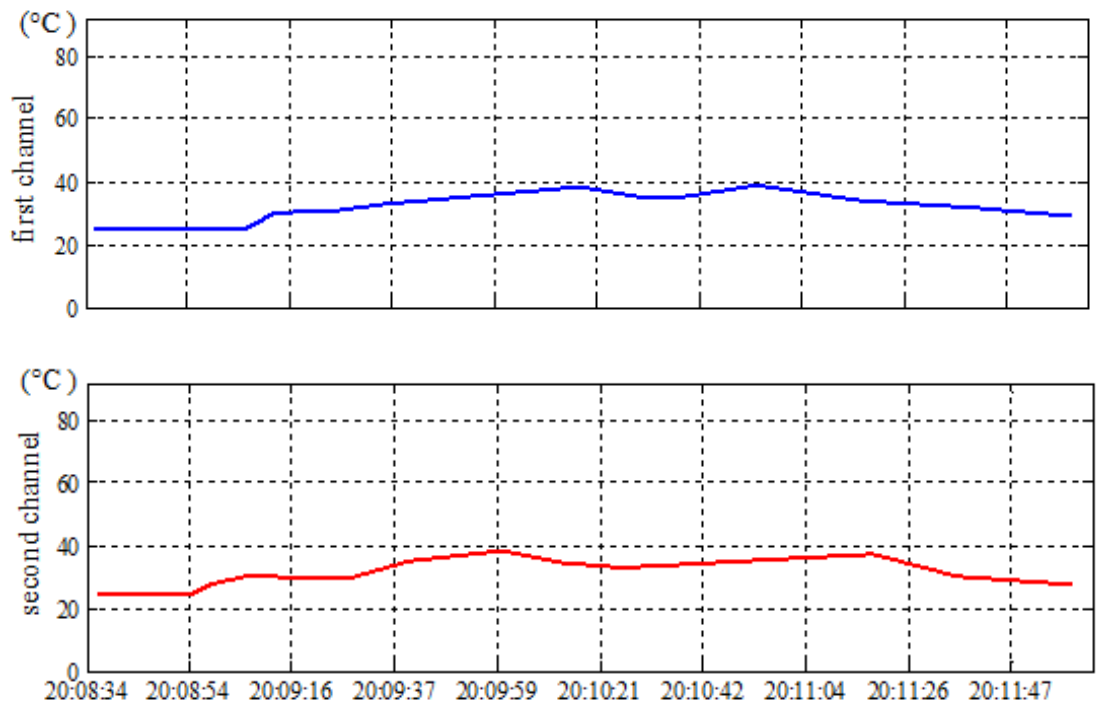

Fig. (7). The historical curve display. 
History curve of the interface you can see in real time the change of each parameter curve is very intuitive, realized dynamic monitoring routine. Fig. (7) shows the historical curve display.

\section{CONCLUSION}

The wireless RF transceiver integrated chip technology is a new wireless communication technology developed in recent years. In recent years, wireless data transmission distance has been the focus of domestic and international development of the field of electronic communication, and has been widely used in the field of home appliances, communications and military in the field of civil. The research content of this paper is to present a variety of wireless communication technology, the main research focuses on the combination of embedded, wireless RF transceiver integrated chips with MPU or DSP based, and combined with the actual use of characteristics of the development of a communication protocol with error correction, calibration and encryption function, to meet the special needs of data transmission in sensor networks.

\section{CONFLICT OF INTEREST}

The author confirms that this article content has no conflict of interest.

\section{ACKNOWLEDGEMENTS}

Declared none.

\section{REFERENCES}

[1] A. Rahimi-Vahed, and A. H. Mirzaei, "A hybrid multi-objective shuffled frog-leaping algorithm for a mixed model assembly line sequencing problem", Computers and Industrial Engineering, vol. 53, no. 4, pp. 642-666, 2007.

[2] X. C. Zhang, X. M. Hu, and G. Z. Cui, "An improved shuffled frog leaping algorithm with cognitive behavior," In: $7^{\text {th }}$ WCICA Conf., pp. 6197-6202, 2008.

[3] T. H. Huynh, "A modified shuffled frog leaping algorithm for optimal tuning of multivariable PID controllers ", In: IEEE International Conference on Industrial Technology, 2008, pp. 1-6.

[4] E. Elbctagi, T. Hegazy, and D. Griemon, "Comparison among five evolutionary-based optimization algorithms", Advanced Engineering Informatics, vol. 19, no. 1, pp. 43-53, 2005.

[5] L. Tong, G. Xu, B. Hassibi, and T. Kailath, "Blind channel estimation based on second-order statistics: a frequency-domain approach," IEEE Transactions on Information Theory, vol. 41, pp. 329334, 1995.

[6] Y. Zhao, and A. Huang, "A novel channel estimation methods for OFDM mobile communication systems based on pilot signals and transform domain processing," In: Proceeding IEEE $47^{\text {th }}$ Vehicular Technology Conference, Phoenix, USA, 1997, pp. 2089-2093.

[7] T. S. Rappaport, "Wireless communications principles and practice," Publishing House of Electronics Industry, 2003.

[8] S. Coleri, M. Ergen, A. Puri, and A. Bahai, "A study of channel in OFDM systems," IEEE VTC, Vancouver, Canada. 2002.

[9] D. B. Van, O. Edfors, and M. Sandle, "On channel estimation in OFDM systems," In: Proceeding IEEE Vehicular Technology Conference, 1999, pp. 815-819.

[10] H. Landau, and H. O. Pollak, "Prolate spheriodal wave functions, Fourier analysis and uncertainty-III: The dimension of the space of essentially time and band-limited signal," Bell System Technical, 2002, pp. 41.

[11] B. Wu; S. Cheng, M. Chen, and H. Wang, "Analysis of decision aided channel estimation in clipped OFDM," Vehicular Technology Conference, vol. 2, pp. 1030-1033, 2005.

Received: May 26, 2015

Revised: July 14, 2015

Accepted: August 10, 2015

(C) Qiuping Wu; Licensee Bentham Open.

This is an open access article licensed under the terms of the (https:/creativecommons.org/licenses/by/4.0/legalcode), which permits unrestricted, noncommercial use, distribution and reproduction in any medium, provided the work is properly cited. 